\title{
RÉPONSES PHYSIOLOGIQUES DE SEPT ESPÈCES DE POISSONS LACUSTRES À UN STRESS DE CAPTURE (FILET MAILLANT ET ÉPUISETTE).
}

\author{
F. BAU, N. FERRONI-CLAVERIE et J.-P. PARENT* \\ UMR - CNRS C 5576, Centre d'Écologie des Systèmes Aquatiques Continentaux, \\ Université Toulouse III, Bat. 4R3, 118 route de Narbonne, \\ 31062 Toulouse Cedex 4, France. \\ * Auteur pour correspondance / Corresponding author
}

\section{RÉSUMÉ}

Les perturbations physiologiques potentiellement liées à la capture des poissons par filet maillant ou par épuisette ont été estimées chez des brochets, sandres, perches, brèmes, chevesnes, carpes et tanches de la retenue hydroélectrique de Pareloup (Aveyron). Toute capture provoque rapidement une hausse significative des taux de catécholamines $\left(>10 \mathrm{ng} \cdot \mathrm{ml}^{-1}\right)$, cortisol $\left(>100 \mathrm{ng} \cdot \mathrm{ml}^{-1}\right)$ et glucose $\left(>1 \mathrm{~g} \cdot \mathrm{l}^{-1}\right)$ et une baisse des taux d'hormones thyroïdiennes $\left(<20 \mathrm{ng} \cdot \mathrm{ml}^{-1}\right)$ du plasma chez les sept espèces. Ces réponses physiologiques au stress de capture apparaissent significativement plus marquées suite à la pêche par filet maillant et ce, notamment, chez les espèces carnassières. D'après ces résultats, l'utilisation de filets maillants s'avère inadéquate pour des recherches à court terme sur la physiologie sanguine de populations naturelles de poissons, soulignant ainsi la nécessité après ce type de pêche d'une mise en place d'infrastructures particulières (e.g., viviers, cages flottantes, etc.) pour la phase ultérieure de récupération physiologique.

Mots-clés : poisson, retenue, physiologie, stress de capture.

\section{PHYSIOLOGICAL RESPONSES OF SEVEN LACUSTRINE FISH SPECIES TO CAPTURE STRESS (GILL NET AND LANDING NET).}

\section{ABSTRACT}

The potential physiological disturbances experienced by fish as a result of capture with gill nets or landing nets were assessed in pike, pikeperch, perch, bream, chub, carp and tench from the hydroelectric reservoir of Pareloup (Aveyron). Capture of fish quickly resulted in both a significant elevation of plasma catecholamine (> $10 \mathrm{ng}^{\mathrm{ml}} \mathrm{f}^{-1}$ ), cortisol (> $100 \mathrm{ng} \cdot \mathrm{ml}^{-1}$ ) and glucose $\left(>1 \mathrm{~g} \cdot \mathrm{l}^{-1}\right.$ ) levels and a drop in plasma thyroid hormone levels $\left(<20 \mathrm{ng} \cdot \mathrm{ml}^{-1}\right)$ in all species. These physiological stress responses were found to be highly increased following gill-netting, especially in carnivorous species. Hence, these data suggest that the use of gill nets is rather inadequate for short-term researches on blood physiology of feral fish populations, then attesting to the necessity of settling up experimental facilities (e.g., fish-ponds, floating coops, etc.) following gill-netting to ensure a complete recovery of fish from capture stress.

Key-words : fish, reservoir, physiology, capture stress. 


\section{INTRODUCTION}

À l'exception de certains travaux menés le plus fréquemment chez des espèces migratrices, il n'y a réellement que peu de recherches sur la physiologie des populations naturelles de poissons. Les études à ce sujet s'avèrent en effet toujours très difficiles car comme cela a été souligné, notamment par LOCHMILLER et al. (1989), CARRAGHER et REES (1994) ou PANKHURST et DEDUAL (1994), elles se heurtent le plus souvent au problème de l'obtention de valeurs cliniques standard de paramètres physiologiques lequel est dû, pour une large part, à la difficulté de minimiser les effets délétères pour l'organisme des procédés de capture. En fait, la prise en compte de ce problème intimement lié aux pratiques courantes en laboratoire ou en aquaculture (e.g., procédés de capture, transport, transfert en bassin, confinement, etc.) est encore actuellement à l'origine de divers travaux visant à tester et à sélectionner, dans un premier temps, les modes de pêche apparemment les moins préjudiciables à l'état physiologique du poisson.

Ainsi, les effets inhérents à une capture par pêche à la ligne ou à la cuiller (e.g., FERGUSON et TUFTS, 1992 ; YOUNGSON et WEBB, 1993 ; PANKHURST et DEDUAL, 1994 ; YOUNG et al., 1994 ; KIEFFER et al., 1995 ; BROBBEL et al., 1996 ; DEDUAL, 1996 ; LOWE et WELLS, 1996 ; WILKIE et al., 1996 ; POTTINGER, 1998), par plongeur sous-marin (e.g., BOLLARD et al., 1993), nasse (e.g., HOPKINS et CECH, 1992), senne (e.g., LEATHERLAND et al., 1989), chalut (e.g., TURUNEN et al., 1994 ; OLLA et al., 1997), tramail (e.g., EGGINTON, 1994 ; CHOPIN et al., 1996) ou pêche électrique (e.g., MESA et SCHRECK, 1989 ; MAULE et MESA, 1994 ; MITTON et MCDONALD, 1994 ; SNYDER, 1995 ; BARTON et GROSH, 1996 ; BARTON et DWYER, 1997 ; MUTH et RUPPERT, 1997) ont-ils été appréciés et quantifiés chez de nombreuses espèces pisciaires par des mesures d'indicateurs classiques de l'état de stress (i.e., catécholamines, cortisol, glucose, lactate ; WEDEMEYER et al., 1990).

L'utilisation de filets maillants, engins de pêche passifs, a été préconisée par I'EIFAC (1975) et citée par BARBIER (1985) pour certaines investigations dans les lacs, réservoirs et milieux potamiques. Ce moyen de capture, ou plus récemment celui des filets maillants verticaux, a montré sa valeur pour les études de recensement (DEGIORGl et al., 1993 ; FLESCH et al., 1995 ; HANSSON et RUDSTAM, 1995) mais s'est avéré également satisfaisant pour une récupération en lacs et rivières d'un grand nombre de poissons dans un état sanitaire correct pour des recherches sur leurs compositions corporelle et tissulaire (NEWSOME et LEDUC, 1975 ; MEDFORD et MACKAY, 1978 ; CAMINADE, 1986 ; SCHWALME et al., 1993 ; FERRONI, 1996 ; ESCORIHUELA et PARENT, 1997).

Par contre, à notre connaissance, ce mode de prélèvement de poissons en milieu naturel n'a fait l'objet d'aucune recherche concernant son impact potentiel sur certaines caractéristiques sanguines. Aussi, nous sommes-nous proposé, dans cette étude, d'évaluer chez des téléostéens lacustres les éventuelles perturbations endocriennes et métaboliques associées à la capture par filet maillant, et de les comparer aux valeurs trouvées chez les mêmes espèces après une rapide prise par épuisette, un procédé de capture communément employé tant en laboratoire qu'en aquaculture.

\section{MATÉRIEL ET MÉTHODES}

\section{Modalités d'échantillonnage}

Un total de 148 poissons issus de sept espèces de téléostéens, i.e., brochet (Esox lucius L.), sandre (Stizostedion lucioperca L.), perche (Perca fluviatilis L.), brème (Abramis brama L.), chevesne (Leuciscus cephalus L.), carpe (Cyprinus carpio L.) et tanche (Tinca tinca L.), a été prélevé au début de l'été 1993 dans la retenue hydroélectrique de Pareloup 
(Aveyron, Massif Central). Au cours des deux campagnes de pêches réalisées en juin, soit juste avant et lors de la phase finale de vidange de cette retenue (Tableau I), deux techniques de capture de poissons différentes ont été utilisées. Le premier lot de 99 individus a été pêché, entre 0 et $10 \mathrm{~m}$ de profondeur, à l'aide de filets monofilament $(30 \times 10 \mathrm{~m})$ de maille $70 \mathrm{~mm}$ posés durant $2 \mathrm{~h}$. Le deuxième lot de 49 individus fut directement récolté à l'épuisette au niveau d'une pêcherie située à $200 \mathrm{~m}$ en aval du barrage. Les poids moyens des sujets sélectionnés restaient comparables entre les deux lots (Tableau II). Pour minimiser le stress dû aux manipulations et à l'émersion, un prélèvement sanguin par ponction intracardiaque (env. 2 à $10 \mathrm{ml}$ par individu) a été rapidement effectué (<3 $\mathrm{min}$ ) après toute capture, en une seule prise sur le poisson maintenu en décubitus dorsal en immersion. Aucun anesthésique n'a été utilisé afin d'éviter tout phénomène d'hémoconcentration. Le sang a été recueilli sur anticoagulant (héparinate de sodium à $75 \pm 25 \mathrm{IU} \mathrm{m}^{-1}$ ), centrifugé pendant $3 \mathrm{~min}$ à $5600 \mathrm{~g}$, et le plasma obtenu congelé dans l'azote liquide et conservé à $-80^{\circ} \mathrm{C}$ pour les analyses différées.

\section{Techniques analytiques}

Après leur extraction du plasma selon le protocole décrit par ATGIÉ et al. (1990), les catécholamines circulantes (adrénaline, noradrénaline et dopamine) ont été mesurées par HPLC à polarité de phase inversée et appariement d'ions couplée à une détection électrochimique, selon la méthode basique de WOODWARD (1982). Les concentrations de cortisol, d'hormones thyroïdiennes (thyroxine' et triiodothyronine) et de glucose du plasma ont été déterminées respectivement par des dosages radioimmunologiques (test Cis Bio International, réf. Cort-ct2), immunoenzymatiques (tests bioMérieux, réf. 69010,69020) et colorimétriques (test bioMérieux, réf. 61271).

\section{Tableau I}

Principales caractéristiques morphométriques et physico-chimiques de la retenue de Pareloup à la date des campagnes de pêches.

\section{Table I}

Main morphometric and physico-chemical characteristics of the reservoir of Pareloup on fishing cruises.

\begin{tabular}{|c|c|c|}
\hline & $\begin{array}{c}1^{\text {ere }} \text { campagne de } \\
\text { pêches }\end{array}$ & $\begin{array}{c}2^{\text {eme }} \text { campagne de } \\
\text { pêches }\end{array}$ \\
\hline $\begin{array}{l}\text { Date } \\
\text { Période }\end{array}$ & $\begin{array}{l}6-8 \text { juin } 1993 \\
\text { avant vidange }\end{array}$ & $\begin{array}{l}14-15 \text { juin } 1993 \\
\text { pendant vidange }\end{array}$ \\
\hline $\begin{array}{l}\text { Caractéristiques morphométriques: } \\
\text { - cote NGF }(\mathrm{m}) \\
\text { - volume de la retenue }\left(10^{6} \mathrm{~m}^{3}\right)\end{array}$ & $\begin{array}{c}779,48 \\
5,42\end{array}$ & $\begin{array}{c}773,76 \\
0,85\end{array}$ \\
\hline $\begin{array}{l}\text { Caractéristiques physico-chimiques : } \\
\text { - température de l'eau }\left({ }^{\circ} \mathrm{C}\right) \\
\text { - oxygène dissous }\left(\mathrm{mg} . .^{-1}\right) \\
\text { - } \mathrm{pH} \\
\text { - } \mathrm{N}-\mathrm{NH}_{4}\left(\mathrm{mg} . \mathrm{I}^{-1}\right) \\
\text { - alcalinité }\left(\mathrm{mgCaCO}_{3} . .^{-1}\right)\end{array}$ & $\begin{array}{c}14-15 \\
8-11 \\
6,8-7,5 \\
\leq 1 \\
21-26\end{array}$ & $\begin{array}{c}12-13 \\
8-11 \\
6,9-7,6 \\
1-2,6 \\
28-32\end{array}$ \\
\hline $\begin{array}{l}\text { Mode de pêche utilisé } \\
\text { Nombre de poissons capturés }\end{array}$ & $\begin{array}{l}\text { filet maillant } \\
\text { lot } 1: 99\end{array}$ & $\begin{array}{l}\text { épuisette } \\
\text { lot } 2: 49\end{array}$ \\
\hline
\end{tabular}




\section{Tableau II}

Poids moyen $(\mathrm{kg})$ et nombre $(\mathrm{n})$ de poissons capturés par espèce dans la retenue de Pareloup par filet maillant (lot 1) et épuisette (lot 2) lors des deux campagnes de pêches de juin 1993. Les valeurs de probabilité $p$ dérivent de tests-t sur échantillons indépendants (cas de variances inégales : $t_{m c}$ ).

\section{Table II}

Mean weight $(\mathbf{k g})$ and number $(\mathrm{n})$ of fish per species caught from the reservoir of Pareloup by gill net (group 1) and landing-net (group 2) during the two fishing cruises of June 1993. Probability values $p$ are given by independent $t$-tests (separate variances test : $t_{m c}$ ).

\begin{tabular}{lcccc}
\hline & lot 1 & lot 2 & $t_{\text {mc }}(\mathrm{ddl})$ & probabilité \\
\hline Brochet & $1,54 \pm 0,44(14)$ & $2,97 \pm 0,64(7)$ & $-1,850(12)$ & $\mathrm{p}=0,089$ \\
Sandre & $2,35 \pm 0,30(15)$ & $2,80 \pm 0,26(7)$ & $-1,119(19)$ & $\mathrm{p}=0,277$ \\
Perche & $0,63 \pm 0,09(21)$ & $0,97 \pm 0,14(7)$ & $-2,080(12)$ & $\mathrm{p}=0,060$ \\
Brème & $1,30 \pm 0,04(24)$ & $1,27 \pm 0,09(7)$ & $0,212(9)$ & $\mathrm{p}=0,837$ \\
Chevesne & $1,38 \pm 0,19(7)$ & $1,85 \pm 0,15(7)$ & $-2,000(11)$ & $\mathrm{p}=0,070$ \\
Carpe & $5,42 \pm 0,86(8)$ & $3,89 \pm 1,97(7)$ & $0,714(8)$ & $\mathrm{p}=0,495$ \\
Tanche & $1,20 \pm 0,07(10)$ & $1,35 \pm 0,23(7)$ & $-0,612(7)$ & $\mathrm{p}=0,559$ \\
\hline
\end{tabular}

\section{Analyses statistiques}

La comparaison statistique des données obtenues pour les deux lots (i.e., prise au filet vs. prise à l'épuisette) a été effectuée en appliquant le test-t de Student sur échantillons indépendants, au seuil de signification fixé à $\alpha=0,05$. Les résultats présentés dans le texte et les tableaux sont exprimés en tant que valeurs moyennes \pm l'erreur standard (S.E.).

\section{RÉSULTATS}

\section{Catécholamines}

Les taux de catécholamines plasmatiques des poissons échantillonnés varient sensiblement suivant la méthode de pêche utilisée (Tableau III). Ainsi les poissons capturés par filet maillant montrent-ils, le plus souvent, des concentrations d'adrénaline (A), noradrénaline (NA) et dopamine (DA) plus élevées que celles mesurées chez les individus récupérés par épuisette au terme de la vidange. Ces différences apparaissent significatives essentiellement chez les trois carnassiers et chez la brème. Les sandres pris au filet présentent les plus forts taux d'adrénaline $\left(98,62 \pm 24,67 \mathrm{ng} \cdot \mathrm{ml}^{-1}\right.$, $\mathrm{n}=15)$ et de noradrénaline $\left(87,99 \pm 20,40 \mathrm{ng} \cdot \mathrm{ml}^{-1}, \mathrm{n}=15\right)$. Bien que l'amplitude de variation des données soit très importante chez cette espèce, l'écart comparé à ceux pris à l'épuisette reste significatif $(81,5$ et $75,6 \%$ pour $A$ et $N A, p<0,01)$. De telles différences significatives entre les deux lots sont également notées chez le brochet $(83,7$ et $83,6 \%$ pour $A$ et $N A, p<0,01)$, la perche $(61,3 \%$ pour $A, p<0,01)$ et la brème $(40,6$ et $60,8 \%$ pour $A$ et $N A, p<0,01)$. Cette dernière affiche aussi les teneurs en dopamine les plus importantes et les plus disparates $\left(9,35 \pm 1,29 \mathrm{ng}_{\mathrm{ml}} \mathrm{m}^{-1}, \mathrm{n}=24\right.$, c.v. $\left.\sim 68 \%\right)$. L'écart entre groupes pour DA est de l'ordre de $95 \%(p<0,001)$ chez cette espèce et de 33, 75 et $77 \%$ chez les sandres, brochets et perches respectivement (Tableau III pour les niveaux de signification). 


\section{Tableau III}

Taux plasmatiques de catécholamines, cortisol, glucose et hormones thyroïdiennes chez sept espèces de téléostéens de la retenue de Pareloup capturées par filet maillant (lot 1) et épuisette (lot 2) lors des deux campagnes de pêches de juin 1993. Voir Tableau II pour les tailles d'échantillonnage. Les valeurs de probabilité $p$ dérivent de tests-t sur échantillons indépendants (cas de variances inégales : $t_{m c}$ ).

\section{Table III}

Plasma contents of catecholamines, cortisol, glucose and thyroid hormones in seven teleost species caught from the reservoir of Pareloup by gill net (group 1) and landing-net (group 2) during the two fishing cruises of June 1993. See Table II for sampling sizes. Probability values $p$ are given by independent $t$-tests (separate variances test : $t_{\mathrm{mc}}$ ).

\begin{tabular}{|c|c|c|c|c|c|c|c|c|c|}
\hline & \multicolumn{5}{|c|}{ Adrénaline $\left(\mathrm{ng} \cdot \mathrm{ml}^{-1}\right)$} & \multicolumn{4}{|c|}{ Noradrénaline $\left(\mathrm{ng} \cdot \mathrm{ml}^{-1}\right)$} \\
\hline & \multicolumn{2}{|c|}{$\operatorname{lot} 1$} & \multicolumn{2}{|c|}{ lot 2} & \multirow{2}{*}{$\begin{array}{c}\text { prob. } \\
p=0,001\end{array}$} & $\operatorname{lot} 1$ & \multicolumn{2}{|l|}{ lot 2} & \multirow{2}{*}{$\begin{array}{c}\text { prob. } \\
p=0,001\end{array}$} \\
\hline \multirow{2}{*}{$\begin{array}{l}\text { Brochet } \\
\text { Sandre }\end{array}$} & $43,31 \pm$ & 8,14 & $7,07 \pm$ & 1,05 & & $37,12 \pm 7,48$ & $6,10 \pm$ & 0,95 & \\
\hline & & & & & $p=0,006$ & $87,99 \pm 20,40$ & & & $p=0,006$ \\
\hline Perche & $9,28 \pm$ & 1,39 & $3,59 \pm$ & 0,87 & $p=0,00$ & $5,60 \pm 1,56$ & $3,98 \pm$ & 0,61 & \\
\hline Brèm & $14,79 \pm$ & 1,44 & $8,78 \pm$ & 1,27 & $p=0,005$ & $25,87 \pm$ & $10,13 \pm$ & 2,68 & \\
\hline Chevesne & $13,59 \pm$ & 1,28 & $19,18 \pm$ & 2,38 & $p=0,067$ & $31,51 \pm 3,53$ & $42,64 \pm$ & 6,23 & $p=0,153$ \\
\hline Carpe & $10,83 \pm$ & 4,39 & $8,87 \pm$ & 4,08 & $p=0,749$ & $12,72 \pm 10,10$ & $12,53 \pm$ & 8,86 & $p=0,989$ \\
\hline Tanche & $12,17 \pm$ & 5,54 & $25,45 \pm 1$ & 11,60 & $p=0,330$ & $12,11 \pm 7,08$ & $31,83 \pm 15$ & 13,60 & $p=0,230$ \\
\hline
\end{tabular}

Dopamine $\left(\mathrm{ng} \cdot \mathrm{ml}^{-1}\right)$

\begin{tabular}{lccc}
\hline & lot 1 & lot 2 & prob. \\
Brochet & $3,38 \pm 0,67$ & $0,85 \pm 0,73$ & $p=0,021$ \\
Sandre & $2,05 \pm 0,22$ & $1,37 \pm 0,10$ & $p=0,010$ \\
Perche & $2,93 \pm 0,41$ & $0,68 \pm 0,32$ & $p=0,000$ \\
Brème & $9,35 \pm 1,29$ & $0,48 \pm 0,18$ & $p=0,000$ \\
Chevesne & $4,18 \pm 1,08$ & $2,81 \pm 0,94$ & $p=0,361$ \\
Carpe & $2,81 \pm 1,12$ & $1,27 \pm 0,44$ & $p=0,236$ \\
Tanche & $3,71 \pm 1,07$ & $0,92 \pm 0,47$ & $p=0,034$
\end{tabular}

Cortisol (ng. $\left.\mathrm{ml}^{-1}\right)$

Glucose $\left(\mathrm{g} . \mathrm{I}^{-1}\right)$

$\begin{array}{cccccc}\text { lot } 1 & \text { lot } 2 & \text { prob. } & \text { lot } 1 & \text { lot } 2 & \text { prob. } \\ 62 \pm 44,4 & 104,06 \pm 37,2 & p=0,003 & 1,38 \pm 0,17 & 0,78 \pm 0,08 & p=0,005 \\ 42 \pm 44,8 & 236,82 \pm 20,6 & p=0,000 & 3,11 \pm 0,36 & 1,29 \pm 0,14 & p=0,000 \\ 50 \pm 39,8 & 300,50 \pm 56,5 & p=0,040 & 2,89 \pm 0,30 & 1,32 \pm 0,09 & p=0,000 \\ 04 \pm 27,7 & 202,73 \pm 60,4 & p=0,001 & 2,18 \pm 0,18 & 1,44 \pm 0,19 & p=0,012 \\ 69 \pm 39,3 & 588,53 \pm 30,7 & p=0,044 & 3,87 \pm 0,51 & 1,35 \pm 0,16 & p=0,002 \\ 65 \pm 104,0 & 181,98 \pm 8,4 & p=0,052 & 2,15 \pm 0,20 & 1,21 \pm 0,31 & p=0,027 \\ 85 \pm 89,1 & 267,29 \pm 53,3 & p=0,520 & 2,74 \pm 0,48 & 1,76 \pm 0,47 & p=0,168\end{array}$

Thyroxine (ng. $\left.\mathrm{ml}^{-1}\right)$

Triiodothyronine $\left(\mathrm{ng} \cdot \mathrm{ml}^{-1}\right)$

\begin{tabular}{lrrcccc}
\hline & lot 1 & \multicolumn{1}{c}{ lot 2} & prob. & lot 1 & lot 2 & prob. \\
Brochet & $9,79 \pm 1,16$ & $19,67 \pm 2,16$ & $p=0,003$ & $2,71 \pm 0,25$ & $3,72 \pm 0,35$ & $p=0,037$ \\
Sandre & $3,45 \pm 1,17$ & $4,99 \pm 0,96$ & $p=0,324$ & $2,35 \pm 0,26$ & $4,83 \pm 0,58$ & $p=0,004$ \\
Perche & $9,25 \pm 1,19$ & $20,35 \pm 2,84$ & $p=0,007$ & $2,00 \pm 0,28$ & $3,25 \pm 0,26$ & $p=0,004$ \\
Brème & $10,48 \pm 0,79$ & $9,65 \pm 0,80$ & $p=0,465$ & $4,12 \pm 0,96$ & $3,08 \pm 0,56$ & $p=0,354$ \\
Chevesne & $10,86 \pm 1,01$ & $8,64 \pm 1,99$ & $p=0,346$ & $1,87 \pm 0,83$ & $0,67 \pm 0,31$ & $p=0,215$ \\
Carpe & $18,98 \pm 5,74$ & $11,63 \pm 2,79$ & $p=0,276$ & $3,86 \pm 2,26$ & $0,84 \pm 0,70$ & $p=0,236$ \\
Tanche & $8,42 \pm 1,30$ & $10,18 \pm 1,17$ & $p=0,330$ & $2,92 \pm 0,32$ & $2,97 \pm 0,34$ & $p=0,915$ \\
\hline
\end{tabular}




\section{Cortisol et glucose}

Chez la plupart des espèces considérées, les concentrations plasmatiques de cortisol et de glucose diffèrent aussi significativement selon le mode de capture employé (Tableau III), ce qui se traduit pour les deux paramètres par des concentrations nettement plus marquées chez les animaux pêchés au filet maillant. Les chevesnes ainsi échantillonnés montrent, en particulier, les plus fortes teneurs en cortisol $\left(701,69 \pm 39,35 \mathrm{ng} \cdot \mathrm{ml}^{-1}, \mathrm{n}=7\right)$ et glucose $\left(3,87 \pm 0,51 \mathrm{~g} \cdot \mathrm{l}^{-1}, \mathrm{n}=7\right)$, soit respectivement une différence d'env. $16 \%(p<0,05)$ et $65 \%(p<0,01)$ comparée aux valeurs mesurées chez les sujets capturés par épuisette. Cette élévation de la cortisolémie inhérente à la prise au filet des animaux s'exprime, chez les autres espèces, par des écarts entre groupes de l'ordre de $66 \%$ chez le brochet, $55 \%$ chez le sandre, 34,5\% chez la perche, $63 \%$ chez la brème, $57 \%$ chez la carpe et $20 \%$ chez la tanche (Tableau III pour les niveaux de signification). Quant à la glycémie, elle diffère d'env. 43, 59, 54, 34, 44 et $36 \%$ pour les mêmes espèces respectivement.

\section{Hormones thyroïdiennes}

L'incidence de la technique de pêche sur les taux d'hormones thyroïdiennes du plasma n'apparaît clairement que chez les trois espèces carnivores (Tableau III). Seules celles-ci présentent effectivement des teneurs plasmatiques en thyroxine (T4) et triiodothyronine (T3) significativement différentes entre les deux lots avec, contrairement aux autres indices physiologiques examinés, des valeurs nettement plus importantes chez les individus pris à l'épuisette que chez ceux pris au filet. Les concentrations maximales de thyroxine sont ainsi rencontrées chez le brochet $\left(19,67 \pm 2,16 \mathrm{ng} \cdot \mathrm{ml}^{-1}, \mathrm{n}=7\right)$ et chez la perche $\left(20,35 \pm 2,84 \mathrm{ng} \cdot \mathrm{ml}^{-1}, \mathrm{n}=7\right)$, et celles de triodothyronine chez le sandre $\left(4,83 \pm 0,58 \mathrm{ng} \cdot \mathrm{ml}^{-1}, \mathrm{n}=7\right)$ récoltés à l'épuisette. Les écarts entre groupes sont alors de $50 \%$ (T4) et $27 \%$ (T3) chez le brochet, de $51 \%$ (T3) chez le sandre, et de $54,5 \%$ (T4) et $38,5 \%$ (T3) chez la perche.

\section{DISCUSSION}

\section{Effets de la capture sur les hormones du stress}

Les concentrations de catécholamines et de cortisol trouvées dans le plasma de plusieurs espèces pisciaires en condition de repos et après divers stress imposés ont fait l'objet de nombreux articles de synthèse (e.g., NILSSON, 1983 ; WEDEMEYER et al., 1990 ; MCDONALD et MILLIGAN, 1992 ; RANDALL et PERRY, 1992). Bien que le nombre d'espèces examinées reste relativement limité (e.g., truite, saumon, anguille, morue, carpe, plie, roussette, lamproie), il est manifeste qu'il y a des différences intra- et inter-spécifiques significatives dans la sécrétion basale de ces hormones qui peuvent être partiellement associées à des disparités dans les contenus du tissu chromaffine et les niveaux d'activité des espèces considérées (MCDONALD et MILLIGAN, 1992 ; RANDALL et PERRY, 1992). Toutefois, si des valeurs standard minimales de $50 \mathrm{ng} \cdot \mathrm{ml}^{-1}$ ont été rapportées pour la cortisolémie du poisson-chat (Heteropneustes fossilis, Bloch) (LAMBA et al., 1983) ou de la carpe (Cyprinus carpio, L.) (HANKE et al., 1993), plus généralement les auteurs s'accordent à considérer des concentrations d'adrénaline, de noradrénaline et de cortisol inférieures à $10 \mathrm{ng} \cdot \mathrm{ml}^{-1}$ comme des valeurs de base typiques pour les poissons. 
De fait, nos résultats démontrent clairement que les deux modes de capture utilisés dans cette étude déclenchent, chez les sept espèces de téléostéens lacustres examinées, des réponses neuro-endocrines qualitativement similaires et quantitativement caractéristiques d'un état de stress important, comme en témoignent les élévations rapides et marquées après les pêches des teneurs moyennes du plasma en adrénaline et noradrénaline (5-100 ng. $\mathrm{ml}^{-1}$ ) et en cortisol (100-750 ng. $\left.\mathrm{ml}^{-1}\right)$. Cependant, bien que communément élevées, les réponses s'avèrent chez la plupart des espèces significativement plus fortes chez les sujets pris au filet maillant en $2 \mathrm{~h}$ de temps que chez ceux rapidement récupérés à l'épuisette (Tableau III). Cette dernière observation s'accorde ainsi aux données rapportées dans la littérature pour des poissons en bassin et spécifiant que, à moins de réduire le temps entre la pêche et le prélèvement de sang à $3 \mathrm{~min}$, toute capture active les sécrétions adrénergiques et interrénaliennes. Par exemple, chez la plie (Pleuronectes platessa, L.) maintenue en stabulation, WHITE et FLETCHER (1989) ont enregistré selon la sévérité et la durée de l'agitation physique dans les filets une hausse de la cortisolémie de 700 à $800 \%$ supérieure à celle des témoins non stressés, soient des valeurs de l'ordre de 600 à $900 \mathrm{ng} \cdot \mathrm{ml}^{-1}$, voire $1100 \mathrm{ng} \cdot \mathrm{ml}^{-1} \mathrm{chez}$ certains individus. Les perturbations physiologiques notées dans notre étude consécutivement à la prise au filet des animaux apparaissent plus particulièrement conséquentes chez les espèces carnassières notamment chez le sandre. Un état asphyxique prononcé a bien été défini chez ce percidé (BAU, 1997) suite à une importante réduction des mouvements buccaux et operculaires provoquée par la rétention singulière des animaux dans les filets, et l'élévation observée (approx. 77-81\%) des quantités d'amines' dans le système circulatoire est vraisemblablement la première réponse hormonale devant déclencher les mécanismes de compensation destinés à supprimer les effets perturbateurs du stress sur le transport de l'oxygène par le sang. Les catécholamines sont effectivement connues pour assurer, dans un premier temps, une oxygénation artérielle adéquate en optimisant les fonctions cardiovasculaire et respiratoire (OLSON, 1992 ; RANDALL et PERRY, 1992 ; PERRY et REID, 1993). Les forts taux de cortisol trouvés chez le sandre $(+55 \%$ ) ou chez le chevesne (considéré comme un cyprinidé actif et piscivore à l'état adulte; MICHEL et OBERDORFF, 1995) reflètent l'hyperactivité sécrétoire de l'interrénal qui classiquement, dans un deuxième temps, renforce la tolérance aux agressions en intensifiant certaines réponses physiologiques suscitées par les catécholamines (PERRY et REID, 1993 ; PAGNOTTA et al., 1994). Par ailleurs, la variabilité significative des taux d'hormones du stress entre les lots ne semble pas devoir être attribuée à une modification de la qualité physico-chimique de l'eau à la date des pêches (Tableau I). L'amplification de la réponse au stress par un accroissement du niveau thermique a été mise en relief, notamment chez la truite (Oncorhynchus mykiss, Walbaum) après une hypoxie modérée (RANDALL et PERRY, 1992) ou un stress de chasse de 5 min (MILLIGAN et al., 1989), mais le faible écart de température enregistré dans cette étude $\left(2-3^{\circ} \mathrm{C}\right)$ ne laisse pas supposer à une contribution importante de ce facteur dans les différences hormonales notées après la capture.

\section{Effets de la capture sur la glycémie}

Les taux de glucose du plasma sont élevés chez le poisson lors du stress, en premier lieu en tant que conséquence des forts taux de catécholamines circulantes, bien que l'implication du cortisol dans les processus glycogénolytiques/néoglucogéniques n'ait pas été rejetée (VAN DER BOON et al., 1991). La teneur en glucose du plasma à un moment donné est fonction de plusieurs facteurs tels que l'âge de l'animal, le régime alimentaire, le moment depuis le dernier repas, la saison, etc. De fait, elle est considérée comme un indice de stress plus équivoque que le cortisol (WEDEMEYER et al., 1990). Cependant, la quantification du glucose plasmatique peut fournir une information complémentaire valable concernant la sévérité et la durée du stress (POTTINGER, 1998). 
Dans l'étude présentée, les effets des deux modes de capture sur les niveaux glycémiques apparaissent qualitativement similaires et aussi prononcés que ceux observés sur les taux de cortisol du plasma (Tableau III). La glycémie des poissons prélevés dépasse très largement la limite des valeurs cliniques standardisées citée dans la littérature (i.e., $1 \mathrm{~g} . \mathrm{I}^{-1}$ ), hormis pour certains pris à l'épuisette pour lesquels des valeurs basales moyennes assez comparables ont été notées, e.g., pour le brochet (obs. $0,78 \pm 0,08$ vs. $0,68-0,90{\mathrm{~g} . \mathrm{I}^{-1}}^{2}$ SCHWALME et MACKAY, 1985) ou la carpe (obs. $1,21 \pm 0,31$ vs. $0,45-1,30{\mathrm{~g} .1^{-1}}$; POTTINGER, 1998). La réponse glycémique est significativement accentuée chez les sujets pêchés par filet maillant, atteignant des valeurs inhabituelles $\left(>3 \mathrm{~g} \cdot \mathrm{l}^{-1}\right.$ ) en comparaison de celles de poissons d'élevage chez le sandre et le chevesne. Ce résultat suggère l'existence de facteurs identiques contrôlant l'élévation de la cortisolémie et de la glycémie lors de perturbations et supporte ainsi l'hypothèse suivant laquelle l'hyperglycémie induite par le stress est aussi, en grande partie, dépendante du cortisol (VAN DER BOON et al., 1991).

\section{Effets de la capture sur les hormones thyroïdiennes}

Certains auteurs ont fait mention de la synergie fréquente entre le cortisol ef les hormones thyroïdiennes (GAPP, 1987 ; WENDELAAR BONGA, 1993), et d'après VIJAYAN et LEATHERLAND (1989), les modifications de l'activite de la thyroide seraient même les meilleurs indices de réponse à une contrainte chronique des poissons. Mais l'interaction du stress sur la fonction thyroïdienne des poissons apparaît résulter en des réponses différentes selon l'espèce considérée et la nature et durée du stimulus appliqué. II s'est avéré que certains stress à court terme tendaient à augmenter la T4 du plasma, avec soit une diminution soit une certaine stabilité de la T3 (PAGNOTTA et al., 1994; REDDY et al., 1995), tandis que d'autres stress brefs (REDDING et al., 1984) ou à plus long terme (VIJAYAN et LEATHERLAND, 1989 ; WENDELAAR BONGA, 1993 ; HOLLOWAY et al., 1994) semblaient induire une baisse des teneurs plasmatiques en T4 ou T3, voire une réduction globale de l'activité thyroïdienne (HIGGS et al., 1982).

Dans notre étude, l'incidence de la technique de pêche sur les taux des deux iodothyronines du plasma se traduit, chez les trois espèces carnassières, par des valeurs significativement réduites chez les spécimens capturés par filet maillant. Ce résultat s'accorde à la théorie émise par certains auteurs (HIGGS et al., 1982 ; REDDING et al., 1984 ; HOLLOWAY et al., 1994) d'une inhibition de l'axe hypothalamo-hypophysothyroïdien par les produits de l'axe hypothalamo-hypophyso-interrénalien. Le stress important provoqué par la prise au filet doit avoir conduit à une rapide régulation de l'axe hypothalamo-hypophyso-thyroïdien, moyen probable par lequel les réserves énergétiques utilisables sont court-circuitées des processus de croissance et rendues disponibles pour subvenir aux besoins métaboliques immédiats et à court terme des animaux (FARBRIDGE et LEATHERLAND, 1992, 1993). Labsence d'écarts significatifs entre les lots pour tous les cyprinidés et la forte variabilité des données chez certains (Tableau III) pourraient témoigner de différences dans les activités locomotrice et alimentaire des individus (FARBRIDGE et LEATHERLAND, 1992 ; PAGNOTTA et al., 1994) au moment de leur capture.

\section{CONCLUSION}

Les taux de catécholamines et de cortisol plasmatiques observés chez la plupart des espèces après la capture, notamment celle par filet maillant, s'avèrent bien en excès de ceux requis pour causer à long terme des effets adverses sérieux sur la croissance, la reproduction et limmunité chez les salmonidés. Particulièrement importantes chez les espèces carnivores, les variations hormonales et l'altération du métabolisme intermédiaire consécutives à la prise au filet pourraient expliquer les forts taux de mortalité observés 
chez ces poissons (e.g., $75 \%$ chez le sandre) lors de manipulations additionnelles comme le transport, le transfert et le confinement des animaux en bassins de récupération. En comparaison, peu de mortalités après le transfert dans d'autres plans d'eau ont été enregistrées pour les animaux rapidement récupérés à l'épuisette au terme de la vidange de la retenue. D'après ces résultats, l'utilisation de filets maillants n'apparait donc pas satisfaisante pour des recherches à court terme sur la physiologie sanguine de populations sauvages de téléostéens. Pour ce type d'études sur milieu lacustre, il serait nécessaire soit de modifier le mode de prélèvement des poissons (e.g., carrelet, nasse, etc.) soit de mettre en place des infrastructures particulières (e.g., viviers, cages flottantes, etc.) pour la phase ultérieure de récupération physiologique.

\section{BIBLIOGRAPHIE}

ATGIÉ C., NIBBELINK M., AMBID L., 1990. Sympathoadrenal activity and hypoglycemia in the hibernating garden dormouse. Physiol. Behav., 48, 783-787.

BARBIER B., 1985. Les techniques de capture. Engins passifs : les filets maillants. In : GERDEAUX D. and BILLARD R. (Eds.), Gestion piscicole des lacs et retenues artificielles, INRA, Paris, 81-90.

BARTON B.A., GROSH R.S., 1996. Effect of AC electroshock on blood features in juvenile rainbow trout. J. Fish Biol., 49, 1330-1333.

BARTON B.A., DWYER W.P., 1997. Physiological stress effects of continuous- and pulsed-DC electroshock on juvenile bull trout. J. Fish Biol., 51, 998-1008.

BAU F., 1997. Estimation de réponses physiologiques à un stress de capture chez divers téléostéens d'un lac de retenue. Thèse de Doctorat, Université Toulouse III, 225 p.

BOLLARD B.A., PANKHURST N.W., WELLS R.M.G., 1993. Effects of artificially elevated plasma cortisol levels on blood parameters in the teleost fish Pagrus auratus (Sparidae). Comp. Biochem. Physiol., 106A, 157-162.

BROBBEL M.A., WILKIE M.P., DAVIDSON K., KIEFFER J.D., BIELAK A.T., TUFTS B.L., 1996. Physiological effects of catch and release angling in Atlantic salmon (Salmo salar) at different stages of freshwater migration. Can. J. Fish. Aquat. Sci., 53, 2036-2043.

CAMINADE V., 1986. Recherches sur l'état nutritionnel de deux espèces de poissons de la retenue de Pareloup (Aveyron) : le gardon (Rutilus rutilus L.) et la perche (Perca fluviatilis L.). Thèse de 3ème cycle, Institut National Polytechnique de Toulouse, $78 \mathrm{p}$.

CARRAGHER J.F., REES C.M., 1994. Primary and secondary stress responses in golden perch, Macquaria ambigua. Comp. Biochem. Physiol., 107A, 49-56.

CHOPIN F.S., ARIMOTO T., INOUE Y., 1996. A comparison of the stress response and mortality of sea bream Pagrus major captured by hook and line and trammel net. Fish. Res., 28, 277-289.

DEDUAL M., 1996. Observed mortality of rainbow trout caught by different angling techniques in Lake Taupo, New Zealand. N. Am. J. Fish. Manage., 16, 357-363.

DEGIORGI F., GUILLARD J., GRANDMOTTET J.P., GERDEAUX D., 1993. Les techniques d'étude de l'ichtyofaune lacustre utilisées en France : bilan et perspectives. Hydroécol. Appl., 5, 27-42.

EGGINTON S., 1994. Stress response in two Antarctic teleosts (Notothenia coriceps Richardson and Chaenocephalus aceratus Lönnberg) following capture and surgery. J. Comp. Physiol., 164B, 482-491.

E.I.F.A.C. (EUROPEAN INLAND FISHERIES ADVISORY COMMISSION), 1975. Symposium on the methodology for the survey, monitoring and appraisal of fishery resources in lakes and large rivers. Panel reviews and relevant papers. EIFAC Tech. Pap., 23, 746 p. 
ESCORIHUELA D., PARENT J.P., 1997. Variations des fractions protéiques musculaires chez quatre téléostéens capturés dans une retenue au cours d'un cycle annuel et lors d'une vidange. Ichtyophysiologica Acta, 20, 53-66.

FARBRIDGE K.J., LEATHERLAND J.F., 1992. Temporal changes in plasma thyroid hormone, growth hormone and free fatty acid concentrations, and hepatic 5'-monodeiodinase activity, lipid and protein content during chronic fasting and refeeding in rainbow trout (Oncorhynchus mykiss). Fish Physiol. Biochem., 10, 245-257.

FARBRIDGE K.J., LEATHERLAND J.F., 1993. Relationship between appetite and plasma thyroid and growth hormone and metabolite concentrations in rainbow trout, Oncorhynchus mykiss. In : KAUSHIK S.J. and LUQUET P. (Eds.), Fish nutrition in practice, INRA, Paris, 279-286.

FERGUSON R.A., TUFTS B.L., 1992. Physiological effects of brief air exposure in exhaustively exercised rainbow trout (Oncorhynchus mykiss) : implications for " catch and release " fisheries. Can. J. Fish. Aquat. Sci., 49, 1157-1162.

FERRONI J.M., 1996. Caractéristiques morphophysiologiques et composition somatique de la perche (Perca fluviatilis L.) et du gardon (Rutilus rutilus L.) dans un lac de retenue : Pareloup (France). Variations saisonnières et relations avec la valeur nutritive du plancton. Thèse de Doctorat, Université Toulouse III, $85 \mathrm{p}$.

FLESCH A., MASSON G., MORETEAU J.C., 1995. Temporal distribution of perch (Perca fluviatilis L.) in a lake-reservoir (Moselle, France) : analysis of catches with vertical gill nets. Hydrobiologia, 300/301, 335-343.

GAPP D.A., 1987. Endocrine and related factors in the control of metabolism in nonmammalian vertebrates. In: CHESTER JONES I., INGLETON P.M. and PHILLIPS J.G. (Eds.), Fundamentals of comparative vertebrate endocrinology, Plenum press, New York, London, 511-660.

HANKE W., ABO HEGAB S., ASSEM H., BERKOWSKY B., GERHARD A., GUPTA O., REITER S., 1993. Mechanisms of hormonal action on osmotic adaptation in teleost fish. In : BRAUNBECK T., HANKE W. and SEGNER H. (Eds.), Fish ecotoxicology and ecophysiology, VCH Verlagsgesellschaft, Weinheim, 315-326.

HANSSON S., RUDSTAM L.G., 1995. Gillnet catches as an estimate of fish abundance: a comparison between vertical gillnet catches and hydroacoustic abundances of Baltic Sea herring (Clupea harengus) and sprat (Sprattus sprattus). Can. J. Fish. Aquat. Sci., 52, 75-83.

HIGGS D.A., FAGERLUND U.H.M., EALES J.G., MCBRIDE J.R., 1982. Application of thyroid and steroid hormones as anabolic agents in fish culture. Comp. Biochem. Physiol, 73B, 143-176.

HOLLOWAY A.C., REDDY P.K., SHERIDAN M.A., LEATHERLAND J.F., 1994. Diurnal rhythms of plasma growth hormone, somatostatin, thyroid hormones, cortisol and glucose concentrations in rainbow trout, Oncorhynchus mykiss, during progressive food deprivation. Biol. Rhythm Res., 25, 415-432.

HOPKINS T.E., CECH J.J.Jr., 1992. Physiological effects of capturing striped bass in gill nets and fyke traps. Trans. Am. Fish. Soc., 121, 819-822.

KIEFFER J.D., KUBACKI M.R., PHELAN F.J., PHILIPP D.P., TUFTS B.L., 1995. Effects of catch-and-release angling on nesting male smallmouth bass. Trans. Am. Fish. Soc., 124, 70-76.

LAMBA V.J., GOSWAMI S.V., SUNDARARAJ B.I., 1983. Circannual and circadian variations in plasma levels of steroids (cortisol, estradiol-17 $\beta$, estrone, and testosterone) correlated with the annual gonadal cycle in the catfish, Heteropneustes fossilis (Bloch). Gen. Comp. Endocrinol., 50, 205-225. 
LEATHERLAND J.F., DOWN N.E., DONALDSON E.M., DYE H.M., 1989. Changes in plasma thyroid hormone levels in pink salmon, Oncorhynchus gorbuscha, during their spawning migration in the Fraser River (Canada). J. Fish Biol., 35, 199-205.

LOCHMILLER R.L., WEICHMAN J.D., ZALE A.V., 1989. Hematological assessment of temperature and oxygen stress in a reservoir population of striped bass (Morone saxatilis). Comp. Biochem. Physiol., 93A, 535-541.

LOWE T.E., WELLS R.M.G., 1996. Primary and secondary stress responses to line capture in the blue mao mao. J. Fish Biol., 49, 287-300.

MAULE A.G., MESA M.G., 1994. Efficacy of electrofishing to assess plasma cortisol concentration in juvenile chinook salmon passing hydroelectric dams on the Columbia River. N. Am. J. Fish. Manage., 14, 334-339.

MCDONALD D.G., MILLIGAN C.L., 1992. Chemical properties of the blood. In : HOAR W.S., RANDALL D.J. and FARRELL A.P. (Eds.), Fish physiology, vol. XIIB, Academic Press, New York, 55-133.

MEDFORD B.A., MACKAY W.C., 1978. Protein and lipid contents of gonads, liver and muscle of Northern pike (Esox lucius) in relation to gonad growth. J. Fish. Res. Board Can., 35, 213-219.

MESA M.G., SCHRECK C.B., 1989. Electrofishing mark-recapture and depletion methodologies evoke behavioural and physiological changes in cutthroat trout. Trans. Am. Fish. Soc., 118, 644-658.

MICHEL P., OBERDORFF T., 1995. Feeding habits of fourteen European freshwater fish species. Cybium, 19, 5-46.

MILLIGAN C.L., GRAHAM M.S., FARRELL A.P., 1989. The response of trout red cells to adrenaline during seasonal acclimation and changes in temperature. J. Fish Biol., $35,229-236$.

MITTON C.J.A., MCDONALD D.G., 1994. Consequences of pulsed DC electrofishing and air exposure to rainbow trout (Oncorhynchus mykiss). Can. J. Fish. Aquat. Sci., 51, 1791-1798.

MUTH R.T., RUPPERT J.B., 1997. Effects of electrofishing fields on captive embryos and larvae of razorback sucker. N. Am. J. Fish. Manage., 17, 160-166.

NEWSOME G.E., LEDUC G., 1975. Seasonal changes of fat content in the yellow perch (Perca flavescens) of two Laurentian lakes. J. Fish. Res. Board Can., 32, 2214-2221.

NILSSON S., 1983. Autonomic nerve function in the vertebrates. In : FARNER D.S., HEINRICH B., JOHANSEN K., LANGER H., NEUWEILER G. and RANDALL D.J. (Eds.), Zoophysiology, vol. XIII., Springer-Verlag, Berlin, Heidelberg, New York, 255 p.

OLLA B.L., DAVIS M.W., SCHRECK C.B., 1997. Effects of simulated trawling on sablefish and walleye pollock : the role of light intensity, net velocity and towing duration. J. Fish. Biol., 50, 1181-1194.

OLSON K.R., 1992. Blood and extracellular fluid volume regulation : role of the reninangiotensin system, kallikrein-kinin system and atrial natriuretic peptides. In : HOAR W.S., RANDALL D.J. and FARRELL A.P. (Eds.), Fish physiology, vol. XIIB, Academic Press, New York, 136-254.

PAGNOTTA A., BROOKS L., MILLIGAN L., 1994. The potential regulatory roles of cortisol in recovery from exhaustive exercise in rainbow trout. Can. J. Zool., 72, 2136-2146.

PANKHURST N.W., DEDUAL M., 1994. Effects of capture and recovery on plasma levels of cortisol, lactate and gonadal steroids in a natural population of rainbow trout. J. Fish Biol., 45, 1013-1025. 
PERRY S.F., REID S.D., 1993. $\beta$-adrenergic signal transduction in fish : interactive effects of catecholamines and cortisol. Fish Physiol. Biochem., 11, 195-203.

POTTINGER T.G., 1998. Changes in blood cortisol, glucose and lactate in carp retained in anglers' keepnets. J. Fish Biol., 53, 728-742.

RANDALL D.J., PERRY S.F., 1992. Catecholamines. In : HOAR W.S., RANDALL D.J. and FARRELL A.P. (Eds.), Fish physiology, vol. XIIB, Academic Press, New York, 255-299.

REDDING J.M., SCHRECK C.B., BIRKS E.K., EWING R.D., 1984. Cortisol and its effects on plasma thyroid hormone and electrolyte concentrations in freshwater and during seawater acclimation in yearling coho salmon, Oncorhynchus kisutch. Gen. Comp. Endocrinol., 56, 146-155.

REDDY P.K., VIJAYAN M.M., LEATHERLAND J.F., MOON T.W., 1995. Does RU486 modify hormonal responses to handling stressor and cortisol treatment in fed and fasted rainbow trout? J. Fish Biol., 46, 341-359.

SCHWALME K., MACKAY W.C., 1985. The influence of exercice-handling stress on blood lactate, acid-base and plasma glucose status of Northern pike (Esox lucius L.). Can. J. Zool., 63, 1125-1129.

SCHWALME K., MACKAY W.C., CLANDININ M.T., 1993. Seasonal dynamics of fatty acid composition in female Northern pike (Esox lucius L.). J. Comp. Physiol., 163B, 277-287.

SNYDER D.E., 1995. Impacts of electrofishing on fish. Fisheries, 20, 26-27.

TURUNEN T., KAKELA A., HYVARINEN H., 1994. Trawling stress and mortality in undersized (<40 cm) brown trout (Salmo trutta L.). Fish. Res., 19, 51-64.

VAN DER BOON J., VAN DEN THILLART G.E.E.J.M., ADDINK A.D.F., 1991. The effects of cortisol administration on intermediary metabolism in teleost fish. Comp. Biochem. Physiol., 100A, 47-53.

VIJAYAN M.M., LEATHERLAND J.F., 1989. Cortisol-induced changes in plasma glucose, protein, and thyroid hormone levels and liver glycogen content of coho salmon (Oncorhynchus kisutch Walbaum). Can. J. Zool., 67, 2746-2750.

WEDEMEYER G.A., BARTON B.A., MCLEAY D.J., 1990. Stress and acclimation. In : SCHRECK C.B. and MOYLE P.B. (Eds.), Methods for fish biology, American Fisheries Society, Bethesda, Maryland, 451-490.

WENDELAAR BONGA S.E., 1993. Endocrinology. In : EVANS D.H. (ed.), The physiology of fishes, CRC Marine Science Series, CRC Press, Boca Raton, Floride, 469-502.

WHITE A., FLETCHER T.C., 1989. The effect of physical disturbance, hypoxia and stress hormones on serum components of the plaice, Pleuronectes platessa L. Comp. Biochem. Physiol., 93A, 455-461.

WILKIE M.P., DAVIDSON K., BROBBEL M.A., KIEFFER J.D., BOOTH R.K., BIELAK A.T., TUFTS B.L., 1996. Physiology and survival of wild Atlantic salmon following angling in warm summer waters. Trans. Am. Fish. Soc., 125, 572-580.

WOODWARD J.J., 1982. Plasma catecholamines in resting rainbow trout, Salmo gairdneri Richardson, by high pressure liquid chromatography. J. Fish Biol., 21, 429-432.

YOUNG G., BROWN C.L., NISHIOKA R.S., FOLMAR L.C., ANDREWS M., CASHMAN J.R., BERN H.A., 1994. Histopathology, blood chemistry, and physiological status of normal and moribund striped bass (Morone saxatilis) involved in summer mortality (" die-off ") in the Sacramento-San Joaquin Delta of California. J. Fish Biol., 44, 491-512.

YOUNGSON A.F., WEBB J.H., 1993. Thyroid hormone levels in Atlantic salmon (Salmo salar) during the return migration from the ocean to spawn. J. Fish Biol., 42, 293-300. 